\title{
Huge intra-thoracic extension of thyroid-an uncommon presentation of ectopic thyroid
}

\begin{abstract}
Background: Intra-thoracic goitres are commonly extension of the cervical thyroid tissue down into the thorax. malignant transformation in ectopic thyroid tissue is rare.

Case report: A 41year old Malay lady referred to HUSM for investigation of a mass in the right upper thorax. On clinical examination, there is reduced air entry at the right upper thorax. There is no enlargement or nodule of the cervical thyroid. She has no signs or symptoms of thyroid hormone imbalance. CT scan of the shows a large intraparenchymal mass. . Intra-operatively, the mass is well-encapsulated, adjacent to the lung parenchyma and separated from the cervical thyroid gland. Histopathological examination confirms that mass is composed of encapsulated nodular thyroid tissue medially and a large lipoma laterally.
\end{abstract}

Conclusion: Management of Intra-Thoracic Extension of Thyroid requires careful planning and anticipating problems prior to the surgery.

Keywords: intra-thoracic goiter, ectopic thyroid, mediastinal, cervical thyroid tissue, thorax
Volume 7 Issue 2 - 2019

Azhar Amir Hamzah,' Ziyadi G,' Azreen Adnan Syazril, ${ }^{2}$ Abdul Kareem, ${ }^{3}$ Amer Hayat Khan, ${ }^{4}$ Kashif Ullah Khan ${ }^{4}$

'Department of Surgery, Universiti Sains Malaysia, Malaysia ${ }^{2}$ Chronic Kidney Disease (CKD) Resource Center, University Science Malaysia, Malaysia

${ }^{3}$ Department of Radiology, Universiti Sains Malaysia, Malaysia ${ }^{4}$ Department of Clinical Pharmacy, Universiti Sains Malaysia, Malaysia

Correspondence: Azhar bin Amir Hamzah, General Surgeon/ Senior Lecturer, Department of Surgery, School of Medical Sciences, Hospital Universiti Sains Malaysia, Kubang Kerian, Kelantan, Malaysia, Email drazhar786@hotmail.com

\section{Introduction}

Intra-thoracic goitres are commonly extension of the cervical thyroid tissue down into the thorax. Rarely, ectopic thyroid tissue gives rise to an intrathoracic goitre ( $<1 \%$ of all mediastinal tumours). ectopic thyroid tissue may be encountered anywhere, this includes from foramen caecum to lower neck. True malignant transformation in ectopic thyroid tissue is rare. In such cases diagnosis is followed after surgical excision of lesion is pathological examined. Current study reports a rare case of intra thoracic lesion of thyroid. ${ }^{1}$

\section{Case report}

A 41year old Malay lady referred to HUSM for investigation of a mass in the right upper thorax. This mass was found incidentally on a routine chest x-ray (Figure $1 \&$ Figure 2). Generally she is in good health. Patient only gave a history of intermittent, mild chest discomfort for duration of five years. Subject denies any history of chest trauma or chronic cough. On clinical examination, there is reduced air entry at the right upper thorax. There is no enlargement or nodule of the cervical thyroid. She has no signs or symptoms of thyroid hormone imbalance.

CT scans of the shows a large intraparenchymal mass (size $10.7 \mathrm{~cm}$ $x 10.3 \mathrm{~cm} \times 8.5 \mathrm{~cm}$ ). This mass is benign in nature and thorax which pushes the trachea to the left. A connection is present between the mass and the right thyroid gland. There is associated collapse consolidation of the lateral segment of middle lobe of the lung Right thoracotomy and excision of the mass was performed. Intra-operatively, the mass is well-encapsulated, adjacent to the lung parenchyma and separated from the cervical thyroid gland. The mass received blood supply from the intrathoracic vessel. This mass was sent for histopathological examination.

Histopathological examination confirms that mass is composed of encapsulated nodular thyroid tissue medially and a large lipoma laterally. There was no evidence of malignancy.

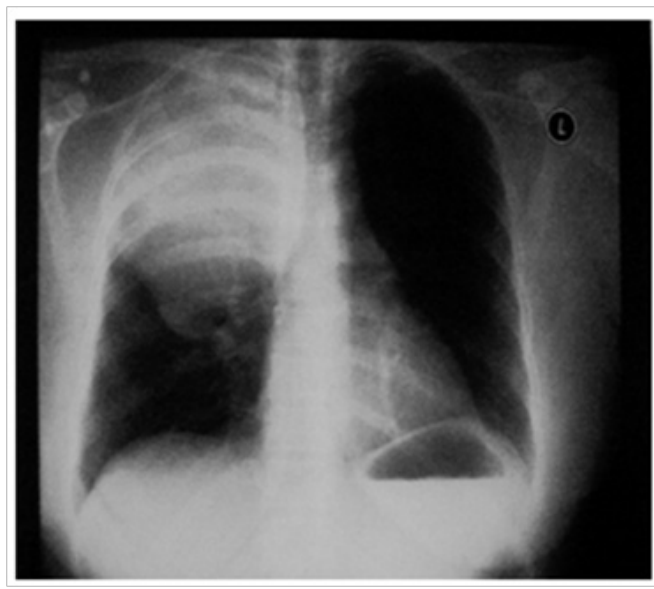

Figure I Initial AP chest X-ray, note the mass occupying the right upper thorax.

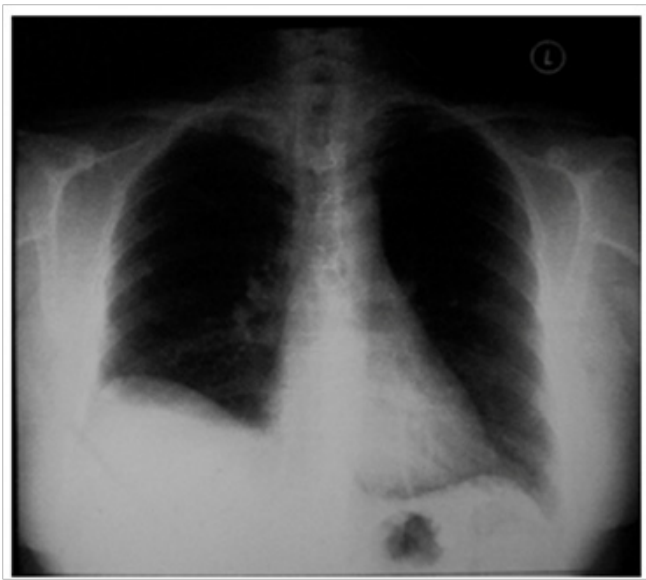

Figure 2 Post-operative chest X-ray. 


\section{Discussion}

Ectopic thyroid tissue has been found from the tongue to the diaphragm. It is commonly found along the course of the thyroglosssal duct. $^{2}$ Ninety percent are found at the base if the tongue (lingual thyroid) and the other $10 \%$ are spread in the anterior aspect of the neck and the mediastinum. ${ }^{3}$ Ectopic intrathoracic thyroid is a rare presentation of thyroid disease, it is defined as the presence of thyroid tissue below the plane of the thoracic inlet. They are mainly locates in the anterior and middle mediastinum. ${ }^{4}$ Intrathoracic thyroid is divided into three types, Small substernal extension of a mainly cervical thyroid goitre, Partial 'intrathoracic goitre', in which the major portion of the goitre is within the thorax, Complete intrathoracic goitre, in which all of the goitre lies within the thoracic cavity. Characteristic of a complete intrathoracic goitre include it derives its blood supply from thoracic vessels, cervical thyroid gland is normal or absent, and the intrathoracic mass if not connected to the cervical thyroid gland except by a thin band of connective tissue.

The pathogenesis of ectopic thyroid is still unclear. It has been postulated that the ectopic thyroid gland is a result of embryological rests which have failed to fuse with the main thyroid tissue during development. $^{3}$ Patients with intrathoracic goitre are usually asymptomatic. Most of the time the tumour is reported as an incidental finding on plain chest $\mathrm{x}$-ray. Other symptoms include cough, dyspnoea, stridor, and dysphagia. These symptoms are typically caused by tracheal and oesophageal compression. ${ }^{4}$ Surgical excision is the definitive treatment; the ectopic intrathoracic thyroid should be removed to rule out malignancy. Thoracotomy or sternotomy is required as total excision of this tissue is usually not possible through a cervical incision.

\section{Conclusion}

Huge Intra-Thoracic Extension of Thyroid could a be difficult and challenging to manage intra operatively, but careful planning and anticipating problems prior to the surgery could a remedy for preventing 'disaster' during the surgery and post operative recovery.

\section{Acknowledgments}

None.

\section{Conflicts of interest}

The authors have no conflicts of interest to disclose.

\section{References}

1. Bhavin C Shah, CS Ravichand, Srinivas Juluri,et al. Ectopic Thyroid Cancer. Ann Thorac Cardiovasc Surg. 2007;13(2):122-124.

2. Mohd Shameem, Rakesh Bhargava, Zuber Ahmad, et al. Intrathoracic Goitre Presenting as Posterior Mediastinal Mass. JIACM 2005;6(4):343344.

3. Shantanu Kumar Sahu, Puneet Kumar Agarwal, Musharraf Husain, et al. Right Supraclavicular Ectopic Thyroid: An Unusual Site of Presentation. The Internet Journal of Surgery. 2007;13(1).

4. Teng Mao, Wentao Fang, A Thomas Pezella. Resection of an Intrathoracic Thyroid Gland. The Internet Journal of Surgery. 2008;4(2). 